\title{
Repeated use of the emergency department: qualitative study of the patient's perspective
}

\author{
M Olsson, H Hansagi
}

\begin{abstract}
Objective-To explore what lies behind repeated emergency department (ED) use, from the patients' own perspectives. Methods-Qualitative study based on in depth interviews with frequent users of the ED at the Huddinge University Hospital, Sweden. Ten adult patients having visited the ED 6-17 times in the previous 12 months were interviewed. The personal meaning they attached to the symptoms and their encounters at the ED were inductively analysed, thereby relating patient behaviour to life conditions.

Results-The frequent ED visitors perceive pain or other symptoms as a threat to life or to personal autonomy. Irrespective of whether or not the patients relate their health problems to a traumatic event, overwhelming anxiety compels them to seek urgent help. Clear cut diagnoses are seldom mentioned. Although none of the patients is homeless or totally lacking in means, the narratives reveal struggles with adverse life circumstances and medical, psychological and/or social problems, including alcohol or other substance misuse. Occasional referrals from the ED to a psychiatrist seem not to lead to any continuous treatment or to a change in the patients' health seeking behaviour. Satisfaction with care becomes adversely affected when the patients perceive that the ED staff classifies their use of the ED as inappropriate or when their symptoms are belittled.

Conclusions-From their own perspectives, frequent ED visitors are in need of urgent care. It is particularly important to these patients that the personal meaning they attach to their symptoms is attended to and respected by the ED staff.

(Emerg Med f 2001;18:430-434)
\end{abstract}

Keywords: emergency department use; qualitative study; patients' perspective

Correspondence to: Dr Olsson, Department of Social Work, B44, Huddinge University Hospital, SE - 141 86 Stockholm, Sweden (mariann.olsson@ socialw.hs.sll.se)

Accepted for publication 11 May 2001

A subgroup of patients makes frequent use of hospital emergency departments (EDs), thereby accounting for a substantial portion of the total number of visits to these facilities. This phenomenon has been reported from many countries, irrespective of the prevailing health care system. ${ }^{1-5}$ At the ED of Huddinge University Hospital, Sweden, some 2000 people ( $4 \%$ of the ED patients) make at least four, in extreme cases up to 70 visits a year, accounting for $18 \%$ of all ED visits. Similar figures have been reported from an Irish hospital ED (3\% of the patients making $12 \%$ of the visits). ${ }^{6}$

Repeated visits may frustrate the staff at busy emergency departments, as these patients' complaints are often judged as non-urgent and inappropriate for ED care, causing prolonged waiting times. ${ }^{71}$ Frequent visitors may also run a risk of fragmented care or of overtreatment because they are seen by many different doctors. ${ }^{12} 13$ This risk may be particularly high in Swedish EDs where emergency medicine is not a distinct specialty, but where doctors from the respective hospital departments staff the ED sections on a rotating basis. The question has been raised whether patients return to the ED because their needs are being met or because their needs are not being met. ${ }^{8}$ One indication of unmet needs might be the fact that those with non-urgent symptoms tend to be less satisfied with ED care than patients with urgent symptoms. ${ }^{14}$

Although quantitative studies on a group level have shown high ED users to have multiple chronic medical and psychosocial problems, ${ }^{1247}$ it has not previously been studied in depth - apart from a single case analysis ${ }^{15}$ what it is that induces these patients to seek frequent, immediate medical attention. When considering this issue, the perspective taken in studies has usually been that of the provider, ${ }^{16}$ or, when focusing on the patient perspective, the methods have been quantitative, comprising record reviews, ${ }^{10}{ }^{12}$ interviewer administered $^{417}$ or self administered ${ }^{2518}$ standardised questionnaires. In this study, we do not test a hypothesis but use a qualitative approach to explore the patients' reasoning behind repeatedly turning to the ED and their experiences of these medical encounters.

\section{Methods}

The study was performed at Huddinge University Hospital, which is located in a suburb of Stockholm, Sweden. The ED of this hospital is staffed with specialists in the field of internal medicine; infectious diseases; general surgery; 
orthopaedics; ear, nose, and throat diseases; gynaecology; neurology; and paediatrics. There is also a psychiatric unit with a separate reception office. Patients are free to visit the ED without prior consultation or referral.

The definition of a frequent visitor, as already used in other studies, ${ }^{257}$ is a patient who has made four or more visits during the previous 12 months. We wanted to include patients who did not require particularly specialised medical care, which is why we used the patient database of the sections for general surgery and internal medicine of the ED to recruit subjects. For two consecutive weeks, 32 adult patients (above the age of 18 years), making visits at any time of day or week were retrospectively identified as frequent visitors. Out of these, a stratified purposeful sampling was made by the variables gender, age, nationality, and number of visits to the ED, in order to ensure variation and enable the identification of patterns. According to Patton, ${ }^{19}$ this sampling method can be used to illustrate characteristics of particular subgroups of interest. Information on the reasons of the index visit was collected from the ED log. Fourteen patients had to be approached in order to obtain a sample of 10 participants - a number we assessed as a minimum to reach variation in individuals, situations and contexts and yet be able to identify common themes. Three of the 14 declined participation and one was too ill psychiatrically.

Qualitative in depth interviews with open questions were carried out. To elicit the patients' narratives, ${ }^{20}$ an interview guide was prepared, covering the following issues: current life circumstances (social situation, social networks and relations, health condition, coping with everyday life), personal history (including events and health changes), health behaviour (smoking, alcohol and drug use, utilisation and experiences of ED and other care sites). The interviews were conducted by two specially trained social workers, not affiliated to the ED, but with experience in psychosocial work with physically ill patients. The sessions took place at the hospital's social work office or in the patient's home and each interview lasted between 1 and 1.5 hours. One interview was carried out with the aid of an interpreter. All subjects agreed to the use of a tape recorder, and the conversations were transcribed verbatim. The Ethics Committee of Huddinge University Hospital gave its approval for the study.

One of the authors of the present paper (MO), reviewed the transcribed narratives, performed the initial structuring, searched the text systematically for main themes, and wrote case summaries in order to maintain a holistic view of each patient in his/her life context. ${ }^{19}$ Data were analysed inductively to identify categories within the areas of interest-subjects' reasoning and interpretation of symptoms connected to care seeking and experiences of care. ${ }^{21}$ Nuances and contradictory or modifying aspects were identified jointly by the authors through systematic comparisons and then discussed with the interviewers. Responsibility for the final analyses and the selection of key themes rests with the two authors of this paper. The participants are assigned fictitious names, from A to J, starting with the oldest person.

\section{Results}

SAMPLE CHARACTERISTICS

In accordance with the sampling procedure, there were equal numbers of men and women among the 10 patients interviewed. Their median age was 51 years (range 23-82). One patient was of foreign nationality. Five patients had made their index visit to the section of general surgery, five to internal medicine. The median number of visits to the ED during the previous year was nine (range 6-17) for patients included from general surgery and eight (range 6-17) for those from the medical section. Their visits, totalling 102 in the past year, covered the ED sections of general surgery, internal medicine, orthopaedics, neurology, urology, and psychiatry. Reasons for the index visit included chest pain (two patients), stomach trouble (two patients), back pain (one patient), headache (one patient), poor general condition (two patients), blood in urine (one patient) and allergy (one patient). The period of time during which the informants made frequent $\mathrm{ED}$ visits varies between one year and 11 years.

In their narratives, most participants talked about how they struggled hard throughout their lives and learned to manage despite scarce resources. Their current social circumstances seem precarious, even though none is homeless or totally lacking in means. All respondents, regardless of age, are either outside or on the periphery of the labour market. Their working lives and means of livelihood have been severely disrupted by their health problems. The majority live alone. Four of the interview subjects feel that they receive good support from their primary social networks. The others describe limited or nonexistent social networks. Seven patients have been in contact with community social services at various periods in their lives. Five patients mention current alcohol and/or drug misuse and another two refer to themselves as former alcoholics. One has a history of overdosing on painkillers.

The interviews reveal a diversity of symptoms and quite a few do not know whether any illness was diagnosed. Only two mention clear cut diagnoses (cardiovascular disease, diabetes). The analyses revealed important common aspects concerning the meanings patients attached to the symptoms, while the experiences of the ED encounters were more varied.

THE MEANING ATTACHED TO SYMPTOMS: THREAT TO LIFE AND TO AUTONOMY

We were struck by the amount of threat and danger that was featured in the narratives. The meaning attached to the symptoms are often given dramatic descriptions by the respondents, indicating the traumatising impact of ill health. Several interview subjects even expressed a fear of dying. The informants' 
personal histories attest to considerable determination and will power. Now, the symptoms pose a threat to the control they exert over their own lives.

The two respondents-Bert and Jennywho suffer from cardiovascular disease and diabetes respectively, feel that their lives are threatened each time severe chest pain or hypoglycaemia occur. "When it becomes unbearable. All you can do is hope that it's not what you think it is... You get angry at yourself for not being how you want to be." (Bert).

Some respondents clearly relate their health problems to a traumatic event. Erik, now divorced and isolated, talked about his episodes of headache as a suffering similar to what he felt five years earlier, when his head was injured as a result of an assault. He is very anxious that the after effects of this injury will eventually lead to his death: "That feeling of impending doom, that fluttery feeling in your chest -I felt I was losing ground, so to speak. It's gone so far that I've even started to leave my door unlocked, so in case someone has to get in, the door will be open ... or else I leave a key with the neighbours so they can get in .... I get twinges in my chest, I was almost dying, I have no one who can sound the alarm or help me, so I went [to the ED]." Fanny relates her pain in the back and her anxiety to a traffic accident 10 years ago, an event jeopardising her marriage and employment. "My legs give way, the pain is unbearable. When I can't walk anymore, when I can't cope with it anymore, that's when I go [to the ED].”

Two respondents talked about seemingly non-urgent and harmless symptoms, not connected to a traumatic event, as if they were life threatening. Doris had not herself been a recent victim of a traumatic event, but she had experienced several traumas in her family. Widowed at the age of 32 with five children, both Doris and several family members have a history of alcohol and substance misuse. Her back aches, and she sees the pain as a serious threat to her life. She also described how eye or stomach symptoms once meant the same threat to her. 'I get so damn angry when I can't do anything, I feel so powerless, you know . . . I guess I'm the nervous type, because I feel there's something wrong with me, I keep looking for what's causing it. Because you don't like to . . .it's not dangerous or anything, but like, it's better that they tell you straight out ... but of course they don't say you have three months to live. Not a single one of them tells you anything. I'm afraid my back will give way." Istvan's friend recently died of cancer and now he fears that the pain he has suffered for the past five months in different parts of the body might have the same consequence. He claims to have a fixed life plan, a plan now seriously threatened by his still undiagnosed pain.

Three of the respondents reported that they had recently been close to giving up and had thoughts of suicide. On the verge of attempting suicide, Hans sought help from his family and found his footing again: "Come hell or high water, now I'm determined to make it..." Fanny had been treated for tablet intoxication, but denies that she intended suicide, rather it was a desperate way to cope with the pain. Erik considered self injury as a way of expressing his desperate situation: "I thought about injuring myself so that I'd be taken in for emergency treatment and they'd have to look after me.... But I didn't want to, not really, not deep down, because I could have hurt myself bad . . . because I want proper follow up."

Only two elderly women did not refer to their conditions as threatening to life or to personal autonomy. Anna, 82 years old, says her family gives her the necessary sense of security.

ENCOUNTERS AT THE ED

When turning to the ED, our informants emphasise that they want their interpretation of the symptoms and their choice to seek urgent help to be respected. However, being in frequent need of urgent medical attention is, for some patients, associated with feelings of inferiority. Fanny has a strong desire to manage the pain on her own. She thus feels ambivalent about seeking care at the ED, but because of unbearable pain, she visits the ED, aware that her own judgement of urgency often differs from that of the ED staff: "I feel ashamed and that I'm not respected . . .. Are you here again?... Fust having to go in causes anxiety. The last time I tried to get someone to go with me, I knew I was in a weak position. I'm so ashamed of myself. But . . . . . when I can't cope with it anymore, that's when I go [to the ED]." Bert has often been afraid of being treated as a "regular" at the emergency department, but he feels that so far he has been well looked after: "They check everything very carefully-they don't just ignore me."

Six respondents, including those with suicidal behaviour, have on some occasion been referred from the ED to a psychiatrist. The encounters at the psychiatric clinic were for all informants connected with an ambiguous outcome and few seem to have received adequate help. They were further referred and/or treatment was discontinued. None has ongoing contact with a psychiatrist, although Istvan is awaiting his first appointment. "They gave me a referral, but she said she wasn't the right person for me, either, but that there was something else... so I postponed it. And so it just fizzled out. I didn't go through with it. I want to put it out of my mind, get rid of all this." (Hans).

The health professionals occasionally assuage the deep worry and console some of the frequent visitors; for others, a perceived lack of empathy increases their distress. Patient satisfaction with care seems to decrease when the ED staff devalue the symptoms presented. "You have to lie there and wait for hours ... and then they put you on a drip. They don't seem to take it so seriously . . .but I don't agree, I think there's something more going on." (George). None of the oldest respondents mentions any unsatisfactory experiences connected with the ED care.

\section{Discussion}

This study suggests that frequent ED visitors have a number of features in common. In the in 
depth interviews, all respondents described stressful life circumstances, with problems of a medical, psychological and/or social character, including own or family members' alcohol and substance misuse. These findings concerning vulnerability are in accord with earlier studies at the group level, albeit in our population not implying homelessness. ${ }^{1-4} 716$ Even when a traumatic event did not precede the problems, the patients appraised pain or other symptoms as a threat to life. The degree of threat and fear can be understood in the context of personal histories and social circumstances. The implications for long term outcome of a perceived threat to life or to body parts has been studied in connection with injury and surgery, ${ }^{22}{ }^{23}$ but to our knowledge it has not previously been related to frequent ED use. Manifestations of psychological distress and the role of psychiatry regarding frequent attenders have recently been discussed in studies of primary care..$^{24}$ Our respondents made strong efforts to cope with the perceived threat and maintain autonomy; however a feeling of powerlessness compelled them to seek help at the ED.

In contrast with younger patients, the oldest patients did not perceive their symptoms as a threat, and they were more satisfied with the way they are looked after at the ED. No gender differences emerged regarding the perceived threat of the symptoms or regarding the use of the ED.

Using in depth interviews helped us to highlight the frequent ED users' motives from their own frames of reference rather than from the view of health professionals. This study, along with qualitative studies in general, used a small sample and statistical representativity was not the goal. Although the method of selecting the sample gave the study breadth regarding patient characteristics and reasons for ED visits, we cannot know how representative our respondents are of frequent $\mathrm{ED}$ users in general, especially not when it comes to other health care systems. The Swedish health care system is fairly comparable to the British one in that patients have good access to primary care. Likewise, groups of general practitioners run out of hours centres. In fact, none of the respondents mentioned lack of access to primary care as the reason for turning to the ED. Furthermore, studies in the USA, where primary care is less accessible, suggest that patients who have access to primary care still use the ED for complaints they believe are serious. ${ }^{5}{ }^{9}$

Our interviews were conducted without immediate connection to a help seeking situation and not in the ED surroundings. This made the interviews reflective and we believe that the narratives were not essentially biased by the fact that some interviews were performed in the patient's home and some in the social worker's office. The interviewers were hospital social workers not informed of the patients' medical reasons for the repeated use. As a result, social circumstances and problems, rather than medical conditions, may have been too much in focus. On the other hand, psychosocial problems might have been even more pronounced among those frequent ED users who could not be interviewed. Furthermore, one retrospective interview, a narrative, may not shed light on the motives for each single ED visit. Nor do we know from this study how the patients actually present their fears and concerns when at the ED. Worries about symptoms, diagnoses and prognoses are often unvoiced in consultations, as demonstrated by a study in general practice. ${ }^{26}$

CAN THE VICIOUS CIRCLE BE BROKEN?

As the complexity of the problems has an adverse effect on social support, a vicious circle may be created. How people present their problems - their "coping stance"-affects the readiness of the potential support providers to assist them. ${ }^{26-28}$ The attitudes of the staff toward the patients are also determined to a large extent by how well they consider the patient to fit in with the kind of care offered by the ED. ${ }^{12}{ }^{15}{ }^{29}$ Thus, there is a risk that the frequent visitors' basic problems will be ignored, as no one will take the time to listen to their stories and discover the personal meaning of the symptoms. ${ }^{11}{ }^{1626}$ This, of course, has a detrimental effect on patient satisfaction.

This study calls for a professional attitude that promotes a sense of security in the frequent visitor. The findings might be used to tailor interventions in order to break the vicious circle of "desperate" visits. Intervention models could for instance include careful interviewing, alcohol advice, invitation to partnership in treatment choice as well as coordination of medical, social and psychiatric care.

We thank the 10 respondents for their narratives. We also thank Dr Sven Andréasson and Professor Valerie DeMarinis for valuable comments on an earlier draft of the paper.

\section{Contributors}

$\mathrm{MO}$ will act as guarantor for the study. $\mathrm{HH}$ and $\mathrm{MO}$ conceived the project. MO prepared the interview guide in consultation with $\mathrm{HH}$, trained the interviewers and made the initial structuring and analysis of the data. $\mathrm{HH}$ and $\mathrm{MO}$ were closely involved in the analyses and interpretation of the results, and wrote the paper. Barbro Carlsson performed the sampling, Marianne Jakobsson and Monica Antser conducted the interviews and took part in discussions of the initial analyses.

Funding: The Swedish Council for Social Research (Project 98-0228:1B)

Conflicts of interest: none.

1 Purdie FRJ, Honigman B, Rosen P. The chronic emergency department patient. Ann Emerg Med 1981;10:298-301.

2 Genell Andrén K. A study of the relationship between social network, perceived ill health and utilization of emergency care. A case-control study. Scand 7 Soc Med 1988;16:87-93.

3 Brown EM, Goel V. Factors related to emergency department use: results from the Ontario Health Survey 1990. Ann Emerg Med 1994;24:1083-91.

4 Lang T, Davido A, Diakité B, et al. Using the hospital emergency department as a regular source of care. Eur $\mathcal{F}$ Epidemiol 1997;13:223-8

5 Lucas RH, Sanford SM. An analysis of frequent users of emergency care at an urban university hospital. Ann Emerg Med 1998;32:563-8

6 Murphy AW, Leonard C, Plunkett PK, et al. Characteristics of attenders and their attendances at an urban accident and emergency department over a one year period. F Accid Emerg Med 1999;16:425-7.

7 Hansagi H, Edhag O, Allebeck P. High consumers of health care in emergency units: how to improve their quality of care. Qual Assur Health Care 1991;3:51-62.

8 Malone RE. Heavy users of emergency services: social construction of a policy problem. Soc Sci Med 1995;40:469-77.

9 Baker DW, Stevens CD, Brook RH. Regular source of ambulatory care and medical care utilization by patients presenting to a public hospital entization fAMA 1994;271:1909-12.

10 Bond TK, Stearns S, Peters M. Analysis of chronic emergency department use. Nurs Econ 1999;17:207-13.

11 Murphy AW. 'Inappropriate' attenders at accident and emergency departments I: definition, incidence, and reasons for attendance. Fam Pract 1998;15:23-32. 
12 Ettinger WH, Casani JA, Coon PJ, et al. Patterns of use of the emergency department by elderly patients. F Gerontol $1987 ; 42 \cdot 638-42$

13 Dale J, Green J, Reid F, et al. Primary care in the accident and emergency department: II. Comparison of general practitioners and hospital doctors. BMF 1995;311:427-30

14 Hansagi H, Carlsson B, Brismar B. The urgency of care need and patient satisfaction at a hospital emergency department. Health Care Manage Rev 1992;17:71-5.

15 Malone RE. Whither the almshouse? Overutilization and the role of the emergency department. F Health Polit Policy Law 1998;23:795-832.

16 Padgett DK, Brodsky B. Psyhosocial factors influencing non-urgent use of the emergency room: a review of the literature and recommendations for research and improved service delivery. Soc Sci Med 1992;35:1189-97.

17 Gill JM, Riley AW. Nonurgent use of hospital emergency departments: urgency from the patient's perspective. $\mathscr{F}$ Fam Pract 1996;42:491-6.

18 Boushy D, Dubinsky I. Primary care physician and patient factors that result in patients seeking emergency care in a 1999;17:405-12.

19 Patton MO. Oualitative evaluation and research methods. Newbury Park: Sage Publications, 1990.

20 Hydén LC. Illness and narrative. Sociol Health Illness 1997;19:48-69.

21 Pope C, Ziebland S, Mays N. Qualitative research in health care. Analysing qualitative data. $B M \mathcal{F} 2000 ; 320: 114-16$.
22 Malt UF. Traumatic effects of accidents. In: Ursano RJ, McCaughey BG, Fullerton CS, eds. The structure of human chaos: individual and community responses to trauma and disaster. Cambridge: Cambridge University Press, 1994

23 Schreiber S, Galai-Gat T. Uncontrolled pain following physical injury as the core-trauma in posttraumatic stress disorder. Pain 1993;54:107-10.

24 Katon W. Panic disorder: relationship to high medical utilization, unexplained physical symptoms, and medical costs. f Clin Psychiatry 1996;57:11-18.

25 Karlsson H, Joukamaa M, Lahti I, et al. Frequent attender profiles: different clinical subgroups among frequent attender patients in primary care. F Psychosom Res 1997;42: 157-66.

26 Barry CA, Bradley CP, Britten N, et al. Patients' unvoiced agendas in general practice consultations: qualitative study. BMF 2000;320:1246-50.

27 Silver RC, Wortman CB, Crofton C. The role of coping in support provision: the self-presentational dilemma of victims of life crises. In: Sarason BR, Sarason IG, Pierce GR, eds. Social support. An interactional view. New York: Wiley, 1990.

28 Olsson M. Social support in bereavement crisis - a study of interaction in crisis situations. Soc Work Health Care 1997; 25:117-30.

29 Dingwall R, Murray T. Categorization in accident departments: 'good' patients, 'bad' patients and 'children'. Sociol Health Illness 1983;5:127-48. 